\title{
J.-M. THOMAS
}

\section{Méthode des éléments finis hybrides duaux pour les problèmes elliptiques du second ordre}

Revue française d'automatique, informatique, recherche opérationnelle. Analyse numérique, tome 10, no 3 (1976), p. 51-79.

<http://www.numdam.org/item?id=M2AN_1976_10_3_51_0>

(C) AFCET, 1976, tous droits réservés.

L'accès aux archives de la revue "Revue française d'automatique, informatique, recherche opérationnelle. Analyse numérique » implique l'accord avec les conditions générales d'utilisation (http://www.numdam.org/legal. php). Toute utilisation commerciale ou impression systématique est constitutive d'une infraction pénale. Toute copie ou impression de ce fichier doit contenir la présente mention de copyright.

\section{Numdam}




\title{
MÉTHODE DES ÉLÉMENTS FINIS HYBRIDES DUAUX POUR LES PROBLEMES ELLIPTIQUES DU SECOND ORDRE
}

\author{
par J.-M. THOMAS \\ Communiqué par P.-A. RaviarT
}

Résumé. - Les méthodes d'approximation conformes basées sur la formulation duale requierent des champs de vecteurs satisfaisant dans chaque sous-domaine l'équation d'équilibre et dont les traces normales sur les interfaces sont réciproques. Nous dualisons cette dernière contrainte à l'aide d'un multiplicateur de Lagrange qui n'est autre que la trace sur les interfaces de la solution du problème primal.

\section{INTRODUCTION}

Pour cette introduction considérons le problème modèle

$$
\left.\begin{array}{rl}
-\Delta u=f & \text { dans } \Omega, \\
u=0 & \text { sur } \partial \Omega,
\end{array}\right\}
$$

où $\Omega$ est un ouvert borné de $\mathbf{R}^{n}$ et où $f$ est une fonction donnée dans l'espace $L_{2}(\Omega)$.

Soit $V^{f}(\Omega)$ la variété affine définie par

$$
V^{f}(\Omega)=\left\{q \in\left(L_{2}(\Omega)\right)^{n} ; \operatorname{div} q+f=0 \text { dans } \Omega\right\} .
$$

Le gradient de la solution $u$ du problème (1.1) est caractérisé comme étant l'unique solution $p$ du problème de minimisation

où $\mathscr{K}$ est la fonctionnelle :

$$
p \in V^{f}(\Omega) ; \quad \mathscr{K}(p)=\operatorname{Inf}_{q \in V^{\prime}(\Omega)} \mathscr{K}(q),
$$

$$
\mathscr{K}(q)=\frac{1}{2} \int_{\Omega}|q|^{2} d x .
$$

En vue d'une approximation numérique de la solution $p$ du problème (1.3), nous découpons le domaine $\Omega$ en sous-domaines $\Omega_{r}$, dans chacun desquels nous pourrons supposer que la fonction $f$ est un polynôme. A cette décomposition du domaine $\Omega$, nous associons la variété affine $X^{\boldsymbol{S}}$ définie par

$$
X^{f}=\left\{q \in\left(L_{2}(\Omega)\right)^{n} ; \operatorname{div} q+f=0 \text { dans chaque } \Omega_{r}\right\} .
$$

(1) Université Paris VI, laboratoire d'Analyse numérique, Tour 55-65.

Revue Française d'Automatique, Informatique et Recherche Opérationnelle, décembre 1976. 
La variété $V^{f}(\Omega)$ est l'ensemble des fonctions $X^{f}$ dont les traces normales sont réciproques sur toute interface de la décomposition du domaine $\Omega$. Il s'avère fort délicat de construire une sous-variété $V_{h}^{f}(\Omega)$ de $V^{f}(\Omega)$ de dimension finie.

Pour éviter cette difficulté, on dualise la contrainte de réciprocité des traces normales à l'aide d'un multiplicateur de Lagrange $\lambda$, qui n'est autre que la trace de la solution $u$ sur le bord des sous-domaines $\Omega_{r}$. Le couple $(p, \lambda)$ est la solution d'un problème de point de selle :

$$
(p, \lambda) \in X^{f} \times M ; \quad \mathscr{L}(p, \lambda)=\operatorname{Inf}_{q \in X^{f}} \operatorname{Sup}_{\mu \in M} \mathscr{L}(q, \mu)
$$

où $M$ est l'espace des multiplicateurs de Lagrange. Il est facile de construire une sous-variété $X_{h}^{f}$ de $\mathbf{R}_{f}$ de dímension finie et un sous-espace $M_{h}$ de $M$ de dimension finie. On cherche alors $\left(p_{h}, \lambda_{h}\right)$ solution du problème

$$
\left(p_{h}, \lambda_{h}\right) \in X_{h}^{f} \times M_{h} ; \quad \mathscr{L}\left(p_{h}, \lambda_{h}\right)=\operatorname{Inf}_{q_{h} \in X_{h}^{f}} \operatorname{Sup}_{\mu_{h} \in M_{h}} \mathscr{L}\left(q_{h}, \mu_{h}\right) .
$$

Nous donnerons des conditions nécessaires et des conditions suffisantes pour que ce problème (1.7) admette une solution et une seule, nous étudierons l'ordre de convergence.

Pour l'interprétation physique de cette méthode ainsi que pour la résolution pratique du système linéaire associé au problème (1.6), nous référons aux articles généraux de Pian [8], Pian et Tong [10] et De Veubeke [5]. Nous généralisons la méthode d'approximation proposée par Pian [9] pour l'étude de la torsion d'une barre élastique; l'étude mathématique de ce problème a été faite par Brezzi [2]. Les résultats de convergence sont obtenus ici à l'aide d'une nouvelle extension des techniques d'éléments finis développées dans Ciarlet [3], Strang et Fix [13] par exemple.

Les résultats sont présentés sur le problème de Dirichlet lié à un opérateur elliptique non nécessairement symétrique. On généralise aisément au cas du problème mêlé avec conditions aux limites de Dirichlet-Neumann non homogènes. Le plan suivi est :

$\S 2$ : Formulations variationnelle duale et hybride duale.

$\S 3$ : Approximation du problème basée sur la formulation hybride duale.

$\S 4$ : Méthode d'éléments finis hybrides duaux.

$\S 5$ : Majorations d'erreur dans la méthode des éléments finis hybrides duaux.

\section{Notations}

L'espace $L_{2}(\Omega)$ est l'espace des fonctions (réelles) de carré sommable pour la mesure de Lebesgue, muni de la norme

$$
\|v\|_{0 . \Omega}=\left\{\int_{\Omega}|v|^{2} d x\right\}^{1 / 2}
$$


Pour tout entier $m \geqq 0, H^{m}(\Omega)$ est l'espace de Sobolev d'ordre $m$ muni de la norme

$$
\|v\|_{m, \Omega}=\left\{\sum_{|\alpha| \leqq m} \int_{\Omega}\left|\frac{\partial^{|\alpha|} v}{\partial x_{1}^{\alpha^{1}} \ldots \partial x_{n}^{\alpha_{n}}}\right|^{2} d x\right\}^{1 / 2},
$$

où $\alpha=\left(\alpha_{1}, \ldots, \alpha_{n}\right)$ est un multi-entier de $\mathbf{N}^{n}$ de longueur $|\alpha|=\alpha_{1}+\ldots+\alpha_{n}$.

Sur cet espace $H^{m}(\Omega)$, nous utiliserons la semi-norme

$$
|v|_{m, \Omega}=\left\{\sum_{|\alpha|=m} \int_{\Omega}\left|\frac{\partial^{m} v}{\partial x_{1}^{\alpha_{1}} \ldots \partial x_{n}^{\alpha_{n}}}\right|^{2} d x\right\}^{1 / 2} .
$$

L'espace $H^{1 / 2}(\Gamma)$ est l'espace des traces sur $\Gamma=\partial \Omega$ des fonctions de $H^{1}(\Omega)$.

L'espace $H^{-1 / 2}(\Gamma)$ est le dual de $H^{1 / 2}(\Gamma)$ lorsque l'espace $L_{2}(\Gamma)$ est identifié à son dual. L'espace $H_{0}^{1}(\Omega)$ est le sous-espace des fonctions de $H^{1}(\Omega)$ à trace nulle sur $\Gamma$.

Si $p$ et $q$ sont deux vecteurs de $\mathbf{R}^{n}$ de composantes respectives $p_{1}, \ldots, p_{n}$ et $q_{1}, \ldots, q_{n}$, on note par $p . q$ leur produit scalaire

$$
p \cdot q=\sum_{i=1}^{n} p_{i} q_{i}
$$

Si $q$ est une fonction de l'espace $\left(H^{m}(\Omega)\right)^{n}$, c'est-à-dire telle que chacune de ses $n$ composantes $q_{i}$ soit une fonction de l'espace $H^{m}(\Omega)$, on note

$$
\|q\|_{m, \Omega}=\left\{\sum_{i=1}^{n}\left\|q_{i}\right\|_{m, \Omega}^{2}\right\}^{1 / 2}
$$

et de même

$$
|q|_{m, \Omega}=\left\{\sum_{i=1}^{n}\left|q_{i}\right|_{m, \Omega}^{2}\right\}^{1 / 2}
$$

\section{FORMULATIONS VARIATIONNELLE DUALE ET HYBRIDE DUALE}

Soit $\Omega$ un ouvert (non vide) borné et connexe de $\mathbf{R}^{n}$, de frontière $\Gamma=\partial \Omega$ lipschitzienne. Soit $\mathscr{A}$ l'opérateur aux dérivées partielles du second ordre défini par

$$
\mathscr{A} u=-\sum_{i, j=1}^{n} \frac{\partial}{\partial x_{i}}\left(a_{i j} \frac{\partial u}{\partial x_{j}}\right)
$$

où les coefficients $a_{i j}$ appartiennent à $L_{\infty}(\Omega)$ et vérifient l'hypothèse d'ellipticité usuelle : il existe une constante $e>0$ telle que

$$
\forall x \in \Omega \text { p.p., } \quad \forall \xi=\left(\xi_{i}\right)_{i=1, \ldots, n} \in \mathbf{R}^{n}, \quad \sum_{i, j=1}^{n} a_{i j}(x) \xi_{j} \xi_{i} \geqq e|\xi|^{2} .
$$


On considère à titre d'exemple le problème de Dirichlet homogène pour l'opérateur $\mathscr{A}$ :

$$
\left.\begin{array}{rlrl}
\mathscr{A} u & =f & \text { dans } \Omega, \\
u=0 & \text { sur } \Gamma,
\end{array}\right\}
$$

où la fonction $f$ est donnée dans l'espace $L_{2}(\Omega)$.

La formulation variationnelle classique - encore appelée formulation variationnelle primale - consiste à trouver $u$ solution du problème :

$$
\forall v \in H_{0}^{1}(\Omega), \quad \int_{\Omega}\left\{\sum_{i, j=1}^{n} a_{i j} \frac{\partial u}{\partial x_{j}} \frac{\partial v}{\partial x_{i}}\right\} d x=\int_{\Omega} f v d x .
$$

Il est bien connu que ce problème (2.3) admet une solution $u$ et une seule. En outre, dans le cas symétrique, c'est-à-dire lorsque $a_{i j}=a_{j i}$ pour tout $i, j=1, \ldots, n$, cette solution $u$ est caractérisée comme étant l'unique élément de $H_{0}^{1}(\Omega)$ qui minimise la fonctionnelle d'énergie.

$$
\mathscr{J}(v)=\frac{1}{2} \int_{\Omega}\left\{\sum_{i, j=1}^{n} a_{i j} \frac{\partial v}{\partial x_{j}} \frac{\partial v}{\partial x_{i}}\right\} \text { \} } \int_{\Omega} f v d x
$$

sur l'espace $H_{0}^{1}(\Omega)$.

\section{Formulation variationnelle duale}

En formulation duale, l'inconnue fondamentale du problème (2.2) n'est plus la fonction $u$ elle-même mais son cogradient (relativement à l'opérateur $\mathscr{A}$ ) c'est-à-dire le vecteur $p$ de composantes

$$
p_{i}=\sum_{j=1}^{n} a_{i j} \frac{\partial u}{\partial x_{j}}
$$

Précisons tout d'abord le cadre fonctionnel utilisé :

Soit $V(\Omega)$ l'espace de Hilbert

$$
V(\Omega)=\left\{q \in\left(L_{2}(\Omega)\right)^{n} ; \operatorname{div} q \in L_{2}(\Omega)\right\}
$$

muni de la norme

$$
\|q\|_{V(\Omega)}=\left\{\|q\|_{0, \Omega}^{2}+\|\operatorname{div} q\|_{0, \Omega}^{2}\right\}^{1 / 2}
$$

Nous utiliserons les résultats d'analyse fonctionnelle suivants ( $c f$. [6] et [7] par exemple) : soit $v$ le vecteur unitaire de la normale à $\Gamma$ dirigé vers l'extérieur de $\Omega$; l'application trace normale $: q \rightarrow v . q$ est linéaire continue surjective 
de l'espace $V(\Omega)$ dans l'espace $H^{-1 / 2}(\Gamma)$. En outre on a la formule de Green :

$$
\left.\begin{array}{c}
\forall q \in V(\Omega), \quad \forall v \in H^{1}(\Omega), \\
\int_{\Omega} v \operatorname{div} q d x+\int_{\Omega} \operatorname{grad} v \cdot q d x=\int_{\Gamma} v v \cdot q d \gamma,
\end{array}\right\}
$$

où au second membre le symbole $\int_{\Gamma} \ldots d \gamma$ désigne la dualité entre les espaces $H^{1 / 2}(\Gamma)$ et $H^{-1 / 2}(\Gamma)$.

A la fonction $f \in L_{2}(\Omega)$, nous associons la variété affine (fermée non vide) de $V(\Omega)$ :

$$
V^{f}(\Omega)=\{q \in V(\Omega) ; \operatorname{div} q+f=0 \text { dans } \Omega\} .
$$

En particulier pour $f=0$, nous avons le sous-espace :

$$
V^{0}(\Omega)=\{q \in V(\Omega) ; \operatorname{div} q=0 \operatorname{dans} \Omega\} .
$$

Il est évident mais fondamental de remarquer

$$
\forall q \in V^{0}(\Omega), \quad\|q\|_{V(\Omega)}=\|q\|_{0, \Omega} .
$$

Avec les hypothèses sur les coefficients $a_{i j}$, la matrice $\left(\left(a_{i j}(x)\right)\right)$ admet un inverse, noté $\left(\left(A_{i j}(x)\right)\right)$, pour presque tout $x$ dans $\Omega$; les fonctions $A_{i j}$ appartiennent à $L_{\infty}(\Omega)$ et il existe une constante $\alpha>0$ telle que

$$
\forall x \in \Omega \text { p. p., } \quad \forall \xi \in \mathbf{R}^{n}, \quad \sum_{i, j=1}^{n} A_{i j}(x) \xi_{j} \xi_{i} \geqq \alpha|\xi|^{2} .
$$

A ces coefficients $A_{i j}$ nous associons la forme bilinéaire $a(p, q)$ définie sur l'espace produit $\left(L_{2}(\Omega)\right)^{n} \times\left(L_{2}(\Omega)\right)^{n}$ par

$$
a(p, q)=\int_{\Omega}\left\{\sum_{i, j=1}^{n} A_{i j} p_{j} q_{i}\right\} d x .
$$

Cette forme bilinéaire est continue sur $\left(L_{2}(\Omega)\right)^{n} \times\left(L_{2}(\Omega)\right)^{n}$ et est $\left(L_{2}(\Omega)\right)^{n}$ elliptique :

$$
\begin{aligned}
\forall p \in\left(L_{2}(\Omega)\right)^{n}, & \forall q \in\left(L_{2}(\Omega)\right)^{n}, \quad a(p, q) \leqq\|a\| \cdot\|p\|_{0, \Omega}\|q\|_{0, \Omega}, \\
& \forall q \in\left(L_{2}(\Omega)\right)^{n}, \quad a(q, q) \geqq \alpha\|q\|_{0, \Omega}^{2} .
\end{aligned}
$$

La formulation variationnelle duale du problème (2.2) consiste à trouver $p$ solution du problème

$$
\left.\begin{array}{c}
p \in V^{f}(\Omega) \\
\forall q \in V^{0}(\Omega), \quad a(p, q)=0 .
\end{array}\right\}
$$

décembre 1976. 
THÉORÈME 2.1 : Le problème (2.13) admet une solution $p=\left(p_{i}\right)_{i=1, \ldots, n}$ et une seule donnée par

$$
p_{i}=\sum_{j=1}^{n} a_{i j} \frac{\partial u}{\partial x_{j}}, \quad i=1, \ldots, n,
$$

où la fonction $u$ est la solution du problème (2.2).

Remarque 2.1 : Dans le cas symétrique, la solution $p$ du problème (2.13) se caractérise comme étant l'unique élément de $V^{f}(\Omega)$ qui minimise la fonctionnelle

$$
\mathscr{K}(q)=\frac{1}{2} a(q, q)
$$

sur la variété affine $V^{f}(\Omega)$. On vérifie aisément

$$
\operatorname{Inf}_{v \in H_{0}^{1}(\Omega)} \mathscr{J}(v)+\operatorname{Inf}_{q \in V^{f}(\Omega)} \mathscr{K}(q)=\mathscr{J}(u)+\mathscr{K}(p)=0 .
$$

Ce résultat justifie l'appellation physique d'énergie complémentaire pour la fonctionnelle $\mathscr{K} \ldots$ relativement à l'énergie $\mathscr{J}$.

Démonstration du théorème 2.1 : La forme bilinéaire $a(p, q)$ est continue sur $V(\Omega) \times V(\Omega)$, d'après (2.11), et elle est $V^{0}(\Omega)$-elliptique, d'après $(2.12)$ et (2.9). Il résulte alors du théorème de Lax-Milgram que le problème (2.13) admet une solution et une seule. Vérifions que la fonction $p$ définie par (2.14) est solution : on a $p \in\left(L_{2}(\Omega)\right)^{n}$ et

$$
\operatorname{div} p+f=-\mathscr{A} u+f=0 \text { dans } \Omega .
$$

Ainsi $p \in V^{f}(\Omega)$. D'autre part pour tout $q \in\left(L_{2}(\Omega)\right)^{n}$, on a

$$
a(p, q)=\int_{\Omega}\left\{\sum_{i, j, k=1}^{n} A_{i j} a_{j k} \frac{\partial u}{\partial x_{k}} q_{i}\right\} d x=\int_{\Omega} \operatorname{grad} u \cdot q d x .
$$

A l'aide de la formule de Green (2.6), on en déduit puisque la solution $u$ appartient à l'espace $H_{0}^{1}(\Omega)$ :

$$
\forall q \in V^{0}(\Omega), \quad a(p, q)=0,
$$

ce qui démontre que les relations $(2.14)$ définissent une solution du problème (2.13).

\section{Formulation hybride duale}

Une telle formulation est liée à une décomposition du domaine. Soit $\bar{\Omega}=\bigcup_{r=1}^{R} \bar{\Omega}_{r}$ une décomposition du domaine $\bar{\Omega}$ en sous-domaines $\bar{\Omega}_{r}$ tels que

(i) $\Omega_{r}$ est une partie ouverte (non vide) de $\Omega$, de frontière $\Gamma_{r}=\partial \Omega_{r}$ lipschitzienne, pour tout $r$; 
(ii) $\Omega_{r} \cap \Omega_{s}=\varnothing$, pour tout $r \neq s$.

On note $v_{r}$ le vecteur unitaire de la normale à $\Gamma_{r}$ dirigé vers l'extérieur de $\Omega_{r}$.

A cette décomposition de $\bar{\Omega}$ nous associons l'espace de Hilbert $X$, isomorphe à l'espace produit $\prod_{r=1}^{R} V\left(\Omega_{r}\right)$ :

$$
X=\left\{q \in\left(L_{2}(\Omega)\right)^{n} ; \operatorname{div} q \in L_{2}\left(\Omega_{r}\right) \text { pour tout } r=1, \quad, R\right\}
$$

muni de la norme

$$
\|q\|_{X}=\left\{\|q\|_{0, \Omega}^{2}+\sum_{r=1}^{\Gamma}\|\operatorname{div} q\|_{0, \Omega_{r}}^{2}\right\}^{1 / 2} .
$$

On a donc

$$
\forall q \in X, \quad\|q\|_{X}^{2}=\sum_{r=1}^{\Gamma}\|q\|_{V\left(\Omega_{r}\right)}^{2} .
$$

A la fonction $f \in L_{2}(\Omega)$, nous associons la variété affine (fermée non vide) de $X$ :

$$
X^{f}=\left\{q \in X ; \operatorname{div} q+f=0 \text { dans } \Omega_{r}, \text { pour tout } r=1, \ldots, R\right\} .
$$

En particulier pour $f=0$, on a le sous-espace

$$
X^{0}=\left\{q \in X ; \operatorname{div} q=0 \text { dans } \Omega_{r} \text {, pour tout } r=1, \ldots, R\right\} .
$$

On remarquera, $c f .(2.9)$,

$$
\forall q \in X^{0}, \quad\|q\|_{X}=\|q\|_{0, \Omega} .
$$

Il est facile de caractériser $V(\Omega)$ comme étant le sous-espace de $X$ des fonctions $q$ telles que

$$
\forall v \in H_{0}^{1}(\Omega), \sum_{r=1}^{r} \int_{\Gamma_{r}} v v_{r} . q d \gamma=0 .
$$

Formellement - l'espace $H^{-1 / 2}\left(\Gamma_{r}\right)$ n'étant pas de type local - cette condition (2.21) exprime la réciprocité des traces normales $v . q$ d'une fonction $q \in V(\Omega)$ sur toute interface intérieure à $\Omega$. Dans la formulation hybride on dualise cette contrainte de réciprocité.

Pour cela on considère l'espace de traces :

$$
\begin{aligned}
& M=\left\{\mu \in \prod_{r=1}^{R} H^{1 / 2}\left(\Gamma_{r}\right) ; \exists v \in H_{0}^{1}(\Omega)\right. \\
& \left.\quad \text { tel que } \mu=v \text { sur tout } \Gamma_{r}, r=1, \ldots, R\right\} .
\end{aligned}
$$

Par définition même de l'espace $M$, pour tout $\mu \in M$ l'ensemble

$$
\mathscr{H}(\mu)=\left\{v \in H_{0}^{1}(\Omega) ; v=\mu \text { sur tout } \mathrm{I}_{r}, r=1, \ldots, R\right\}
$$

décembre 1976. 
est une variété affine fermée non vide de $H_{0}^{1}(\Omega)$. Muni de la norme

$$
\|\mu\|_{M}=\operatorname{Inf}_{n \in \mathscr{H}(\mu)}|v|_{1, \Omega},
$$

l'espace $M$ est un espace de Hilbert.

Sur l'espace $H^{1 / 2}\left(\Gamma_{r}\right)$ nous introduisons la semi-norme

$$
|\mu|_{1 / 2, \Gamma_{r}}=\operatorname{Inf}_{v \in \mathscr{H}_{r}(\mu)}|v|_{1, \Omega_{r}},
$$

où $\mathscr{H}_{r}(\mu)$ est la variété

$$
\mathscr{H}_{r}(\mu)=\left\{v \in H^{1}\left(\Omega_{r}\right) ; v=\mu \text { sur } \Gamma_{r}\right\} .
$$

On pourra remarquer que si $\mathscr{R} \mu$ est le relèvement harmonique de $\mu$ dans $\Omega_{r}$, c'est-à-dire la fonction telle que

on a

$$
\Delta(\mathscr{R} \mu)=0 \quad \text { dans } \Omega_{r}, \quad \mathscr{R} \mu=\mu \quad \text { sur } \Gamma_{r},
$$

$$
|\mu|_{1 / 2, \Gamma_{r}}=|\mathscr{R} \mu|_{1, \Omega_{r}} .
$$

LEMME 2.1: On $a$

$$
\forall \mu \in M, \quad\|\mu\|_{M}^{2}=\sum_{r=1}^{R}|\mu|_{1 / 2, \Gamma_{r}}^{2} .
$$

Démonstration : Soit $v \in \mathscr{H}(\mu)$; il est évident que la restriction $\left.v\right|_{\Omega_{r}}$ de $v$ à $\Omega_{r}$ appartient à la variété $\mathscr{H}_{r}(\mu)$ pour tout $r=1, \ldots, R$. Réciproquement soit $\left(v_{r}\right)_{r=1, \ldots, R} \in \prod_{r=1}^{R} \mathscr{H}_{r}(\mu)$; on construit la fonction $v$ de $L_{2}(\Omega)$ définie par $\left.v\right|_{\Omega_{r}}=v_{r}$ pour tout $r=1, \ldots, R$. Lorsque $\mu \in M$, cette fonction $v$ est dans $H_{0}^{1}(\Omega)$, donc $v \in \mathscr{H}(\mu)$. Cela prouve

$$
\forall \mu \in M, \quad \mathscr{H}(\mu) \simeq \prod_{r=1}^{R} \mathscr{H}_{r}(\mu)
$$

Le résultat $(2,27)$ en résulte trivialement.

On introduit la forme bilinéaire $b(q, \mu)$ définie sur $X \times M$ :

$$
b(q, \mu)=-\sum_{r=1}^{R} \int_{\Gamma r} \mu v_{r} . q d \gamma .
$$

Par application de la formule de Green (2.6) dans chaque $\Omega_{r}$, on obtient pour tout $v \in \mathscr{H}(\mu)$ :

$$
b(q, \mu)=-\int_{\Omega} \operatorname{grad} v \cdot q d x-\sum_{r=1}^{R} \int_{\Omega_{r}} v \operatorname{djv} q d x \leqq \sum_{r=1}^{R}\|q\|_{V\left(\Omega_{r}\right)}\|v\|_{1, \Omega_{r}}
$$

d'où à l'aide de (2.17) et de l'égalité de Cauchy-Schwarz :

$$
b(q, \mu) \leqq\|q\|_{X}\|v\|_{1, \Omega} .
$$


A l'aide de (2.24) et de l'inégalité de Poincaré-Friedrichs, on en déduit l'existence d'une constante $\|b\|$ telle que

$$
\forall q \in X, \quad \forall \mu \in M, \quad b(q, \mu) \leqq\|b\|\|q\|_{X}\|\mu\|_{M} .
$$

Plus précisément, lorsque $q \in X^{0}$, on a pour tout $v \in \mathscr{H}(\mu)$ :

d'où

$$
b(q, \mu)=-\int_{\Omega} \operatorname{grad} v \cdot q d x \leqq\|q\|_{0, \Omega}|v|_{1, \Omega},
$$

$$
\forall q \in X^{0}, \quad \forall \mu \in M, \quad b(q, \mu) \leqq\|q\|_{X}\|\mu\|_{M} .
$$

Choisissant, pour $\mu$ fixé dans l'espace $M$, la fonction $v$ de $\mathscr{H}(\mu)$ harmonique dans chaque $\Omega_{r}$ et la fonction $q=-\operatorname{grad} v$, on obtient en utilisant $(2.25 \mathrm{bis})$ :

$$
\forall \mu \in M, \quad \exists q \in X^{0}, \quad b(q, \mu)=\|\mu\|_{M}^{2}=\|q\|_{X}\|\mu\|_{M} .
$$

On démontre ainsi

$$
\forall \mu \in M, \operatorname{Sup}_{q \in X^{0}} \frac{b(q, \mu)}{\|q\|_{X}}=\|\mu\|_{M} .
$$

La formulation hybride duale consiste à trouver un couple $(p, \lambda)$ solution du problème :

$$
\left.\begin{array}{c}
(p, \lambda) \in X^{f} \times M, \\
\forall q \in X^{0}, \quad a(p, q)+b(q, \lambda)=0, \\
\forall \mu \in M, \quad b(p, \mu) \quad=0 .
\end{array}\right\}
$$

THÉORÈME 2.2 : Le problème (2.31) admet une solution $(p, \lambda)$ et une seule donnée par

$$
\begin{gathered}
p_{i}=\sum_{j=1}^{n} a_{i j} \frac{\partial u}{\partial x_{j}} \text { dans } \Omega, \quad i=1, \ldots, n, \\
\lambda=u \text { sur } \Gamma_{r}, \quad r=1, \ldots, R,
\end{gathered}
$$

où $u$ est la solution du problème (2.2).

Remarque 2.2 : Dans le cas symétrique $\left(a_{i j}=a_{i j}\right)$, la solution $(p, \lambda)$ du problème (2.31) se caractérise comme l'unique point de selle de la fonctionnelle

$$
\mathscr{L}(q, \mu)=\frac{1}{2} a(q, q)+b(q, \mu)
$$

sur l'espace produit $X^{f} \times M$. Ainsi $\lambda=u$ apparaît comme le multiplicateur de Lagrange associé aux contraintes de réciprocité des traces normales de $p$ à travers toute interface intérieure de la décomposition de $\bar{\Omega}$ en sousdomaines.

décembre 1976. 
Démonstration du théorème 2.2 : Par linéarité le problème (2.31) admet au plus une solution si le problème homogène associé

$$
\begin{gathered}
(p, \lambda) \in X^{0} \times M, \\
\forall y \in X^{0}, \quad a(p, q)+b(q, \lambda)=0, \\
\forall \mu \in M, \quad b(p, \mu)
\end{gathered}
$$

admet pour unique solution $p=0, \lambda=0$. Soit $(p, \lambda)$ solution de (2.34). On a

$$
a(p, p)=0 \text {, }
$$

d'où $p=0$ en utilisant la $X^{0}$-ellipticité de la forme bilinéaire $a(p, q)$ $[c f .(2.12)$ et $(2.20)]$; par suite $\lambda$ vérifie

$$
\forall q \in X^{0}, \quad b(q, \lambda)=0 .
$$

A l'aide de $(2.30)$, on en déduit $\lambda=0$. La solution du problème $(2.31)$ est unique.

Pour démontrer l'existence d'une solution, il suffit de vérifier que le couple $(p, \lambda)$ donné en $(2.32)$ et $(2.33)$ fournit une solution. D'après le théorème 2.1 , on a $p \in V^{f}(\Omega)$. Par suite $p \in X^{f}$ et satisfait la deuxième équation de (2.31) :

$$
\forall \mu \in M, \quad b(p, \mu)=0 .
$$

D'autre part puisque $u \in H_{0}^{1}(\Omega)$, la fonction $\lambda$ appartient à l'espace $M$.

Enfin en utilisant la formule de Green (2.6) dans chaque $\Omega_{r}$, on a pour tout $q \in X:$

$$
a(p, q)+b(q, \lambda)=\int_{\Omega} \operatorname{grad} u \cdot q d x-\sum_{r=1}^{R} \int_{\Gamma_{r}} u v_{r} \cdot q d \gamma=\sum_{r=1}^{R} \int_{\Omega_{r}} u \operatorname{div} q d x
$$

Ainsi le couple $(p, \lambda)$ satisfait la première équation de $(2.31)$ :

$$
\forall q \in X^{0}, \quad a(p, q)+b(q, \lambda)=0 .
$$

REMARQue 2.3 : On aurait pu démontrer directement l'existence et l'unicité du problème (2.31) en utilisant un résultat de F. Brézzi ([1], th. 1.1).

REMARQUe 2.4 : On a les caractérisations

$$
\begin{aligned}
& V^{0}(\Omega)=\left\{q \in X^{0} ; \forall \mu \in M, b(q, \mu)=0\right\}, \\
& V^{f}(\Omega)=\left\{q \in X^{f} ; \forall \mu \in M, b(q, \mu) 0\right\} .
\end{aligned}
$$

\section{APPROXIMATION DU PROBLÈME BASEE SUR LA FORMULATION HYBRIDE} DUALE

Nous supposons $\left(^{1}\right)$ connaître explicitement a priori un élément $\tilde{p}$ de $X^{f}: \bar{p} \in\left(L_{2}(\Omega)\right)^{n}$ et

$$
\operatorname{div} \bar{p}+f=0 \text { dans } L_{2}\left(\Omega_{r}\right) \text { pour tout } r=1, \ldots, R .
$$

(1) Nous reviendrons sur cette hypothèse à la fin du paragraphe 4. 
La variété affine $X^{f}$ est le translaté du sous-espace $X^{0}$ par la fonction $\bar{p}$ :

$$
X^{f}=\vec{p}+X^{0} .
$$

Ainsi la formulation hybride duale (2.31) consiste à trouver le couple $(\tilde{p}, \lambda)$ solution de

$$
\begin{aligned}
(\tilde{p}, \lambda) \in X^{0} \times M, & \\
\forall q \in X^{0}, \quad a(\tilde{p}, q)+b(q, \lambda) & =-a(p, q), \\
\forall \mu \in M, \quad b(\tilde{p}, \mu) \quad & =-b(\vec{p}, \mu)
\end{aligned} \mid
$$

où nous avons posé

$$
p=p+\tilde{p}
$$

Pour définir une méthode d'approximation interne du problème (3.3), nous nous donnons

(i) un sous-espace $X_{h}^{0}$ de $X^{0}$ de dimension finie;

(ii) un sous-espace $M_{h}$ de $M$ de dimension finie.

On considère alors le problème : trouver $\left(\tilde{p}_{h}, \lambda_{h}\right)$ solution de

$$
\left.\begin{array}{rl}
\left(\tilde{p}_{h}, \lambda_{h}\right) \in X_{h}^{0} \times M_{h}, & \\
\forall q_{h} \in X_{h}^{0}, \quad a\left(\tilde{p}_{h}, q_{h}\right)+b\left(q_{h}, \lambda_{h}\right) & =-a\left(p, q_{h}\right) \\
\forall \mu_{h} \in M_{h}, \quad b\left(\tilde{p}_{h}, \mu_{h}\right) & =-b\left(\bar{p}, \mu_{h}\right) .
\end{array}\right\}
$$

En vue de l'étude théorique, il est agréable d'introduire la variété affine $X_{h}^{f}$ translatée du sous-espace $X_{h}^{0}$ par la fonction $\vec{p}$ :

$$
X_{h}^{f}=\bar{p}+X_{h}^{0}
$$

et de poser

$$
p_{h}=\bar{p}+\tilde{p}_{h} .
$$

Ainsi le problème (3.5) consiste à trouver $\left(p_{h}, \lambda_{h}\right)$ solution de

$$
\left.\begin{array}{c}
\left(p_{h}, \lambda_{h}\right) \in X_{h}^{f} \times M_{h}, \\
\forall q_{h} \in X_{h}^{0}, \quad a\left(p_{h}, q_{h}\right)+b\left(q_{h}, \lambda_{h}\right)=0, \\
\forall \mu_{h} \in M_{h}, \quad b\left(p_{h}, \mu_{h}\right) \quad=0 .
\end{array}\right)
$$

On obtient là une méthode d'approximation du problème basée sur la formulation hybride duale (2.31).

Guidé par la remarque 2.4, il est naturel de définir les espaces

$$
\begin{aligned}
& V_{h}^{0}(\Omega)=\left\{q_{h} \in X_{h}^{0} ; \forall \mu_{h} \in M_{h}, b\left(q_{h}, \mu_{h}\right)=0\right\}, \\
& V_{h}^{f}(\Omega)=\left\{q_{h} \in_{\varepsilon} X_{h}^{f} ; \forall \mu_{h} \in M_{h}, b\left(q_{h}, \mu_{h}\right)=0\right\},
\end{aligned}
$$

décembre 1976. 
Alors si $\left(p_{h}, \lambda_{h}\right)$ est solution du problème (3.8), $p_{h}$ est solution du problème

$$
\begin{gathered}
p_{h} \in V_{h}^{f}, \\
\forall q_{h} \in V_{h}^{0}, \quad a\left(p_{h}, q_{h}\right)=0.1
\end{gathered}
$$

Nous avons là une méthode d'approximation du problème basée sur la formulation duale (2.13).

THÉORÈME 3.1 : Le problème (3.8) admet une solution $\left(p_{h}, \lambda_{h}\right)$ et une seule si et seulement si les espaces $X_{h}^{0}$ et $M_{h}$ satisfont l'hypothèse de compatibilité :

$$
\left\{\mu_{h} \in M_{h} ; \forall q_{h} \in X_{h}^{0}, b\left(q_{h}, \mu_{h}\right)=0\right\}=\{0\} .
$$

En outre si $\left(p_{h}, \lambda_{h}\right)$ est solution de (3.8), alors $p_{h}$ est l'unique solution du prohlème (3.11).

Remarque 3.1 : Nous verrons dans les exemples que lorsque la condition (3.12) est satisfaite, alors $V_{h}^{0}(\Omega)$ n'est pas un sous-espace de $V^{0}(\Omega)$; on obtient donc en (3.13) une méthode d'approximation externe du problème $(2.13)$.

Remarque 3.2 : Dans le cas symétrique $\left(a_{i j}=a_{i j}\right)$, lorsque la condition (3.12) est satisfaite, la solution $\left(p_{h}, \lambda_{h}\right)$ du problème (3.8) se caractérise comme l'unique point de selle de la fonctionnelle

$$
\mathscr{L}\left(q_{h}, \mu_{h}\right)=\frac{1}{2} a\left(q_{h}, q_{h}\right)+b\left(q_{h}, \mu_{h}\right)
$$

sur l'espace produit $X_{h}^{f} \times M_{h}$.

Démonstration du théorème 3.1 : Par construction le problème (3.8) se ramène à la résolution d'un système linéaire carré d'ordre $\operatorname{dim}\left(X_{h}^{0}\right)+\operatorname{dim}\left(M_{h}\right)$. Ce problème (3.8) admet donc une solution et une seule si et seulement si le problème homogène associé admet une solution unique : utilisant la $X^{0}$-elliptticité de la forme bilinéaire $a(p, q)$, on a l'unicité de $P_{h}$; la condition (3.19) est équivalente à l'unicité de $\lambda_{h}$.

La seconde partie de ce théorème 3.1 est évidente; notons que l'existence d'une solution $\left(p_{h}, \lambda_{h}\right)$ du problème (3.8) assure que la variété affine $V_{h}^{f}(\Omega)$ est non vide.

Majorations D'ERREUR : Considérant $h$ comme un paramètre $>0$ destiné à tendre vers zéro, nous appelons « constante indépendante de $h$ " toute constante indépendante du choix de la décomposition de $\bar{\Omega}$ en sous-domaines et du choix des sous-espaces $X_{h}^{0}$ et $M_{h}$.

Avant d'énoncer le résultat fondamental, remarquons que l'hypothèse de compatibilité (3.12) signifie que l'application

$$
\left.\mu_{h} \rightarrow\left\|\mu_{h}\right\|\right|_{h} ^{\star}=\operatorname{Sup}_{q_{h} \in X_{h}^{0}} \frac{b\left(q_{h}, \mu_{h}\right)}{\left\|q_{h}\right\|_{X}}
$$


est une norme sur $M_{h}$. Nous dirons que les espaces $X_{h}^{0}$ et $M_{h}$ satisfont une hypothèse de compatibilité uniforme si les normes $\left\|\mu_{h}\right\|_{h}^{\star}$ et $\left\|\mu_{h}\right\|_{M}$ sont uniformément (en $h$ ) équivalentes sur l'espace $M_{h}$.

Comme $X_{h}^{0}$ est un sous-espace de $X^{0}$, on a d'après (2.30) :

$$
\forall \mu_{h} \in M_{h}, \quad\left\|\mu_{h}\right\|_{h}^{\star} \leqq\left\|\mu_{h}\right\|_{M} .
$$

L'hypothèse de compatibilité uniforme signifie donc l'existence d'une constante $\beta>0$, indépendante de $h$, telle que

$$
\forall \mu_{h} \in M_{h}, \operatorname{Sup}_{q_{h} \in X_{h}^{0}} \frac{b\left(q_{h}, \mu_{h}\right)}{\left\|q_{h}\right\|_{x}} \geqq \beta\left\|\mu_{h}\right\|_{M} .
$$

THÉORÈME 3.2 : On suppose que les espaces $X_{h}^{0}$ et $M_{h}$ satisfont l'hypothèse de compatibilité uniforme (3.13). Alors le problème (3.8) admet une solution $\left(p_{h}, \lambda_{h}\right)$ et une seule et il existe une constante $C$ indépendante de $h$ telle que

$\left\|p-p_{h}\right\|_{X}+\left\|\lambda-\lambda_{h}\right\|_{M} \leqq C\left\{\operatorname{Inf}_{q_{h} \in X_{h}^{f}}\left\|p-q_{h}\right\|_{X}+\operatorname{Inf}_{\mu_{h} \in M_{h}}\left\|\lambda-\mu_{h}\right\|_{M}\right\}$

où $(p, \lambda)$ est la solution du problème (2.31).

$\mathrm{Ce}$ résultat est une application immédiate d'un théorème fondamental dû à $\mathrm{F}$. Brézzi ([1], th. 2.1). Dans notre problème particulier, la constante $C$ intervenant dans (3.14) ne dépend que des constantes $\|a\|, \alpha$ et $\beta$ définies respectivement en (2.11), (2.12) et (3.13).

Avec la fonction $\tilde{p}$ définie par (3.4), on a

$$
\operatorname{Inf}_{q_{h} \in X_{h}^{f}}\left\|p-q_{h}\right\|_{X}=\operatorname{Inf}_{\tilde{q}^{h} \in X_{h}^{0}}\left\|\tilde{p}-q_{h}\right\|_{X}=\operatorname{Inf}_{\tilde{q}_{h} \in X_{h}^{0}}\left\|\tilde{p}-\tilde{q}_{h}\right\|_{0, \Omega}
$$

Ainsi d'après (3.14) l'étude de l'erreur commise sur la solution se ramène à l'étude des erreurs d'approximation commises sur $\tilde{p}$ et $\lambda$. La principale difficulté consiste à vérifier sur les exemples l'hypothèse de compatibilité uniforme (3.13). Pour des raisons purement techniques, ce problème ne sera abordé qu'en dimension d'espace $n=2$.

\section{MÉTHODE D'ÉLÉMENTS FINIS HYBRIDES DUAUX}

Nous appliquons les résultats généraux développés au paragraphe précédent dans le cas où $\Omega$ est un ouvert polygonal de $\mathbf{R}^{2}$ muni d'une triangulation $\mathscr{T}_{h}$ à l'aide de triangles $K$ de diamètre $\leqq h$.

$$
\bar{\Omega}=\bigcup_{K \in \mathscr{T}_{h}} K .
$$

Les définitions (2.19) et (2.22) des espaces $X^{0}$ et $M$ s'écrivent à présent

$$
\begin{gathered}
X^{0}=\left\{q \in\left(L_{2}(\Omega)\right)^{2} ; \forall K \in \mathscr{T}_{h}, \operatorname{div} q=0 \text { dans } K\right\} \\
M=\left\{\mu \in \prod_{K \in \mathscr{T}_{h}} H^{1 / 2}(\partial K) ; \exists v \in H_{0}^{1}(\Omega)\right. \\
\text { tel que } \left.\mu=v \text { sur tout } \partial K, K \in \mathscr{T}_{h}\right\} .
\end{gathered}
$$

décembre 1976. 
Soit $m$ un entier $\geqq 1$; on choisit pour sous-espace $M_{h}$ de $M$ :

$$
M_{h}=\left\{\mu_{h} \in M ; \forall K \in \mathscr{T}_{h},\left.\mu_{h}\right|_{\partial K} \in P_{m}(\partial K)\right\},
$$

où $P_{m}(\partial K)$ est l'espace des restrictions au bord $\partial K$ des polynômes à deux variables de degré $\leqq m$. Cet espace $P_{m}(\partial K)$ est encore l'ensemble des fonctions définies sur le bord $\partial K$, polynômiales de degré $\leqq m$ sur chaque côté du triangle $K$ et qui sont continues aux trois sommets de ce triangle. Pour tout $\mu_{h} \in M_{h}$ il existe une fonction $v \in \mathscr{H}\left(\mu_{h}\right)$ telle que

$$
v \in H_{0}^{1}(\Omega) ; \quad \forall K \in \mathscr{T}_{h},\left.\quad v\right|_{K} \in P_{m}(K)
$$

(dès que $m \geqq 3$, il n'y a pas unicité d'une telle fonction $v$ ). On pourra donc prendre pour degrés de liberté des fonctions de $M_{h}$ les mêmes degrés de liberté que dans l'approximation classique de type " serendipity d'ordre $m$ " du problème (2.3).

Soient d'autre part $k$ et $k^{\prime}$ deux entiers satisfaisant $0 \leqq k \leqq k^{\prime}$. On se donne pour tout triangle $K \in \mathscr{T}_{h}$ un espace $\mathscr{P}(K)$ de fonctions définies sur $K$ et à valeurs dans $\mathbf{R}^{2}$ tel que

$$
\left(P_{k}(K)\right)^{2} \subset \mathscr{P}(K) \subset\left(P_{k \prime}(K)\right)^{2} .
$$

On choisit alors pour sous-espace $X_{h}^{0}$ de $X^{0}$ :

$$
X_{h}^{0}=\left\{q_{h} \in X^{0} ; \forall K \in \mathscr{T}_{h},\left.q_{h}\right|_{K} \in \mathscr{P}(K)\right\} .
$$

A titre d'exemple lorsque $k=k^{\prime}=0, X^{0}$ est l'espace des fonctions $q$ à valeurs dans $\mathbf{R}^{2}$ constante dans chaque triangle $K$ de $\mathscr{T}_{h}$, la condition div $q=0$ dans $K$ étant automatiquement vérifiée. Lorsque $k=k^{\prime}=1, X_{h}^{0}$ est l'espace des fonctions $q=\left(q_{1}, q_{2}\right)$ qui sont dans chaque triangle $K$ de $\mathscr{T}_{h}$ de la forme

$$
\left\{\begin{array}{l}
q_{1}=\alpha_{01}+\alpha_{11} x_{1}+\alpha_{21} x_{2} \\
q_{2}=\alpha_{02}+\alpha_{12} x+\alpha_{22} x_{2} .
\end{array} \text { avec } \alpha_{11}+\alpha_{22}=0 .\right.
$$

D'après le théorème 3.1 , le problème $(3.8)$ associé à ces choix (4.4) du sous-espace $M_{h}$ et (4.6) du sous-espace $X_{h}^{0}$ admet une solution et une seule si l'hypothèse de compatibilité (3.12) est satisfaite. Introduisant la notation

$$
Z_{h}=\left\{\mu_{h} \in M_{h} ; \forall q_{h} \in X_{h}^{0}, \sum_{K \in \mathscr{T}_{h}} \int_{\partial K} \mu_{h} v_{K} \cdot q_{h} d \gamma=0\right\},
$$

cette hypothèse de compatibilité s'écrit brièvement

$$
Z_{h}=\{0\} .
$$

THÉORÈME 4.1 : Une condition nécessaire pour que l'hypothèse de compatibilité (3.12) soit satisfaite est

$$
k^{\prime} \geqq m-1 .
$$


Démonstration: Soit $\left\{A_{i}\right\}_{i \in I}$ l'ensemble des sommets de la triangulation et soit $A_{i_{0}} \notin \Gamma$. On définit une fonction $\mu_{0} \in M$ de la manière suivante :

Sur chaque côté $A_{i_{0}} A_{j}, \mu_{0}$ est la fonction polynômiale de degré $k^{\prime}+2$ telle que

$$
\left\{\begin{array}{l}
\forall v \in P_{k^{\prime}}, \quad \int_{A i_{0} A_{j}} \mu_{0} v d \gamma=0, \\
\mu_{0}\left(A_{i_{0}}\right)=1, \quad \mu_{0}\left(A_{j}\right)=0 .
\end{array}\right.
$$

et sur tout autre côté $A_{i} A_{j}\left(i \neq i_{0}, j \neq i_{0}\right), \mu_{0}=0$.

La fonction $\mu_{0}$ ainsi construite est un élément non nul de l'espace $M$ qui satisfait :

$$
\forall q_{h} \in X_{h}^{0}, \quad \sum_{K \in \mathscr{T}_{h}} \int_{\partial K} \mu_{0} v_{K} \cdot q_{h} d \gamma=0
$$

Or si la condition (4.9) n'est pas satisfaite, c'est-à-dire si $k^{\prime} \leqq m-2$, cette fonction $\mu_{0}$ appartient à l'espace $M_{h}$ donc au sous-espace $Z_{h}$, ce qui démontre le théorème 4.1 .

Avant d'énoncer une condition suffisante pour que l'hypothèse de compatibilité soit satisfaite, nous étudions les espaces

$$
Z_{h}(\partial K)=\left\{\mu \in P_{m}(\partial K), \forall q \in \mathscr{P}(K) \cap V^{0}(K), \int_{\partial K} \mu v_{K} \cdot q d \gamma=0\right\} .
$$

LEMME 4.1 : Si $k \geqq m, m$ pair, ou si $k \geqq m-1, m$ impair, on a

$$
Z_{h}(\partial K)=P_{0}(\partial K) \text {. }
$$

Lorsque $k=k^{\prime}=m-1, m$ pair, l'espace $Z_{h}(\partial K)$ est de dimension 2.

Démonstration : Il suffit de vérifier ces résultats lorsque $\mathscr{P}(K)=\left(P_{k}(K)\right)^{2}$, ce que nous supposerons au cours de cette démonstration. Notons qu'alors $q \in \mathscr{P}(K) \cap V^{0}(K)$ si et seulement s'il existe une fonction $w \in P_{k+1}(K)$ telle que $q=\operatorname{rot} w$; la trace normale $v_{K}$. $q$ est l'opposée de la dérivée tangentielle $\partial w / \partial \tau_{K}$ prise le long du bord $\partial K$ orienté dans le sens direct :

$$
\int_{\partial K} \mu v_{K} \cdot q d \gamma=-\int_{\partial K} \mu \frac{\partial w}{\partial \tau_{K}} d \gamma=\int_{\partial K} \frac{d \mu}{d \tau_{K}} w d \gamma
$$

Ainsi on peut écrire

$$
Z_{h}(\partial K)=\left\{\mu \in P_{m}(\partial K) ; \forall \in P_{k+1}(\partial K), \int_{\partial K} \frac{d \mu}{d \tau_{K}} w d \gamma=0\right\} .
$$

décembre 1976. 
Notant $S_{m-1}(\partial K)$ l'espace des fonctions polynomiales de degré $\leqq m-1$ sur chaque côté du triangle $K$, toute fonction $\mu$ de $Z_{h}(\partial K)$ satisfait

$$
\frac{d \mu}{d \tau_{K}} \in S_{m-1}(\partial K) ; \quad \forall w \in P_{k+1}(\partial K), \quad \int_{\partial K} \frac{d \mu}{d \tau_{K}} w d \gamma=0 .
$$

On en déduit (la démonstration de ce point est donnée dans [12]) :

- si $k \geqq m, m$ pair, ou si $k \geqq m-1, m$ impair, on a

$$
\frac{d \mu}{d \tau_{K}}=0
$$

- si $k=m-1, m$ pair, il existe une constante $c_{K}^{\prime}$ telle que

$$
\frac{d \mu}{d \tau_{K}}=c_{K}^{\prime} \theta_{\partial K}
$$

où $\theta_{\partial K}$ est la fonction de $S_{m-1}(\partial K)$ qui s'annule aux $m-1$ points de GaussLobatto de chaque côté du triangle $K$ et telle que $\left({ }^{2}\right)$ :

$$
\theta_{\partial K}\left(A_{i+}\right)=+1 \quad \text { pour } i=1,2,3,
$$

où $A_{1}, A_{2}, A_{3}$ (et $A_{4}=A_{1}$ ) est une numérotation des sommets du triangle $K$ en parcourant le bord $\partial K$ dans le sens direct.

Puisque $\theta_{\partial K}$ s'annule aux points de Gauss-Labatto, on a

$$
\int_{A_{i} A_{i+1}} \theta_{\partial K} d \gamma=0, \quad i=1,2,3
$$

et par conséquent il existe une primitive $\Theta_{\partial K}$ de $\theta_{\partial K}$ telle que

$$
\frac{d \Theta_{\partial K}}{d \tau_{K}}=\theta_{\partial K} ; \quad \Theta_{\partial K}\left(A_{i}\right)=0, \quad i=1,2,3 .
$$

Il est clair que cette primitive $\Theta_{\partial K}$ appartient à l'espace $P_{m}(\partial K)$.

Par intégration, on déduit de (4.13) que l'appartenance d'une fonction $\mu$ à l'espace $Z_{h}(\partial K)$ équivaut à

- si $k \geqq m, m$ pair, ou si $k \geqq m-1, m$ impair, il existe une constante $c_{K}$ telle que $\mu=c_{K}$;

- si $k=m-1, m$ pair, il existe deux constantes $c_{K}$ et $c_{K}^{\prime}$ telles que $\mu=c_{K}+c_{K}^{\prime} \Theta_{\partial K}$

REMARQue 4.1 : La démonstration précédente prouve en outre que dans le cas $k=m-1, m$ pair, il suffit, pour que l'espace $Z_{h}(\partial K)$ soit réduit

( $\left.{ }^{2}\right)$ La notation $\theta_{\partial K}\left(A_{i_{+}}\right)$[resp. $\theta_{\partial K}\left(A_{i_{-}}\right)$] désigne la valeur en $A_{i}$ de la fonction polynômiale $\theta_{\partial K}$ sur le côté $A_{i} A_{i+1}$ (resp. $\left.A_{i-1} A_{t}\right)$. Puisque $m-1$ est impair, on a $\theta_{\partial K}\left(A_{i-}\right)=-1$. 
à $P_{0}(\partial K)$, que l'espace $\mathscr{P}(K)$ contienne outre $\left(P_{k}(K)\right)^{2}$ une fonction $q_{L, K}=\operatorname{rot} w_{L, K}$ telle que

$$
\int_{\partial K} \theta_{\partial K} w_{L, K} d \gamma \neq 0
$$

On trouvera dans [12] une détermination explicite d'une telle fonction $w_{L, K}$ appartenant à $P_{k+2}(K)$.

L'importance de cette remarque n'apparaîtra que lors des majorations d'erreur.

THÉORÈME 4.2 : Une condition suffisante pour que l'hypothèse de compatibilité (3.12) soit satisfaite est

$$
k \geqq m-1 .
$$

COROllaire 4.1 : Si $k=k^{\prime}$, c'est-à-dire si $\mathscr{P}(K)=\left(P_{k}(K)\right)^{2}$, la condition (4.14) est une condition nécessaire et suffisante pour que l'hypothèse de compatibilité (3.12) soit satisfaite.

Démonstration du théorème 4.2: Soit $\mu_{h}$ un élément quelconque de $Z_{h}$. Pour tout triangle $K \in \mathscr{T}_{h}$, la restriction $\mu_{h: \partial K}$ de $\mu_{h}$ à $\partial K$ appartient à $Z_{h}(\partial K)$. D'après le lemme 4.1 , lorsque la condition (4.14) est satisfaite, il existe deux constantes $c_{K}$ et $c_{K}^{\prime}$ telles que

$$
\forall K \in \mathscr{T}_{h}, \quad \mu_{h ! \partial K}=c_{K}+c_{K}^{\prime} \Theta_{\partial K},
$$

où la fonction $\Theta_{\partial K}$ a été explicitée au cours de la démonstration du lemme 4.1; la constante $c_{K}^{\prime}$ est nulle si $k \geqq m, m$ pair, ou si $k \geqq m-1, m$ impair.

Exprimons à présent que la fonction $\mu_{h}$ appartient à l'espace $M$ : pour tous triangles $K_{1}$ et $K_{2}$ adjacents, les restrictions $\mu_{h^{\prime} \partial K_{1}}$ et $\mu_{h ! O K_{2}}$ définissent la même fonction sur le côté commun $\left(\partial K_{1}\right) \cap\left(\partial K_{2}\right)$ d'où par connexité

$$
\forall K_{1}, K_{2} \in \mathscr{T}_{h}, \quad c_{K_{1}}=c_{K_{2}}, \quad c_{K_{1}}^{\prime}=c_{K_{2}}^{\prime} .
$$

D'autre part puisque $\mu_{h}=0$ sur la frontière $\Gamma$, on a pour tout triangle $K_{0}$ ayant un côté inclus dans $\Gamma$

On en déduit

$$
c_{K_{0}}=0, \quad c_{K_{0}}^{\prime}=0 .
$$

$$
\forall K \in \mathscr{T}_{h}, \quad c_{K}=0 ; \quad c_{K}^{\prime}=0,
$$

soit $\mu_{h}=0$, ce qui prouve que la condition (4.8) est satisfaite.

Le résultat suivant prouve que la méthode des éléments finis hybrides duaux peut s'interpréter comme une approximation externe du problème (2.13).

Proposition 4.1 : Lorsque la condition (4.14) est satisfaite, $V_{h}^{0}(\Omega) n^{\prime}$ 'est pas un sous-espace de $V^{0}(\Omega)$. 
Démonstration: Soit $K_{0}$ un triangle frontalier de $\mathscr{T}_{h}$, de sommets $A_{1}, A_{2}$ et $A_{3}$; on suppose le côté $A_{2} A_{3}$ inclus dans la frontière $\Gamma$ et $A_{1} \notin \Gamma$. On définit alors une fonction $q_{0} \in X^{0}$ de la manière suivante :

$q_{0}=0$ dans tout triangle $K$ distinct de $K_{0}$ :

$q_{0}=\operatorname{rot} w_{0}$, dans le triangle $K_{0}$, où la fonction $w_{0}$ est un polynôme de $P_{m}\left(K_{0}\right)$ tel que :

si $m$ est impair,

$\left\{\begin{array}{l}w_{0}\left(A_{1}\right)=+1, w_{0}=0 \text { sur le côté } A_{2} A_{3}, \\ \partial w_{0} / \partial \tau_{K o}=0 \text { en les }(m-1) \text { points de Gauss-Lobatto de chaque côté } A_{1} A_{2} \\ \text { et } A_{1} A_{3} ;\end{array}\right.$

et si $m$ est pair,

$$
\left\{\begin{array}{l}
w_{0}\left(A_{1}\right)=0, w_{0}=0 \text { sur le côté } A_{2} A_{3}, \\
\partial w_{0} / \partial \tau_{K_{0}}=0 \text { en les }(m-1) \text { points de Gauss-Lobatto de chaque côté } A_{1} A_{2} \\
\quad \text { et } A_{1} A_{3}, \\
\left.w_{0}\left(A_{12}\right)=w_{0}\left(A_{13}\right)=1, A_{12}\left(\text { resp. } A_{13}\right) \text { milieu de } A_{1} A_{2} \text { (resp. } A_{1} A_{3}\right) .
\end{array}\right.
$$

(On démontre l'existence de telles fonctions $w_{0}$; la trace de $w_{0}$ sur le bord $\partial K_{0}$ est définie de manière unique.)

On vérifie facilement que pour toute fonction $\mu$ appartenant à $P_{m}\left(\partial K_{0}\right)$ et nulle sur le côté $A_{2} A_{3}$, on a

$$
\int_{\partial K_{0}} \mu v_{K_{0}} \cdot q_{0} d \gamma=-\int_{\partial K_{0}} \mu \frac{\partial w_{0}}{\partial \tau_{K_{0}}} d \gamma=0
$$

Par suite :

$$
\forall \mu_{h} \in M_{h}, \quad b\left(q_{0}, \mu_{h}\right)=0 .
$$

Lorsque la condition (4.14) est satisfaite, la fonction $q_{0}$ appartient ainsi au sous-espace $V_{h}^{0}(\Omega)$. Or la trace normale de $q_{0}$ sur le côté $A_{1} A_{2}$ (de même que sur le côté $A_{1} A_{3}$ ) est discontinue : div $q_{0}$ pris au sens des distributions dans $\Omega$ ne définit pas une fonction de $L_{2}(\Omega)$; ainsi $q_{0}$ n'appartient pas au sous-espace $V^{0}(\Omega)$.

En conclusion de ce paragraphe, il résulte que la condition (4.14) est une condition suffisante pour que le problème (3.8), associé aux choix (4.4) et (4.6), admette une solution $\left(p_{h}, \lambda_{h}\right)$ et une seule. Toutes hypothèses de régularité faites, nous allons voir que cette condition (4.14) n'est pas en général une condition suffisante pour obtenir les majorations d'erreur optimales auxquelles il semble naturel de s'attendre.

D'autre part il n'est guère réaliste de supposer connu a priori un élément $\bar{p}$ de la variété $X^{f}$. Nous déterminerons explicitement un relèvement $p_{h}$ de $f_{h}$, 
i. e. $\vec{p}_{h} \in X^{f_{h}}$, où $f_{h}$ est la fonction de $L_{2}(\Omega)$ donc la restriction à tout triangle $K \in \mathscr{T}_{h}$ est la projection orthogonale de $f$ sur $P_{m-1}(K)$ :

$$
f_{h \mid K} \in P_{m-1}(K) ; \quad \vee v \in P_{m-1}(K), \quad \int_{K} f v d x=\int_{K} f_{k} v d x
$$

On est ainsi conduit à résoudre le problème

$$
\left.\begin{array}{c}
\left(p_{h}, \lambda_{h}\right) \in X_{h}^{f_{h}} \times M_{h}, \\
\forall q_{h} \in X_{h}^{0}, \quad a\left(p_{h}, q_{h}\right)+b\left(q_{h}, \lambda_{h}\right)=0, \\
\forall \mu_{h} \in M_{h}, \quad b\left(p_{h}, \mu_{h}\right) \quad=0,
\end{array}\right\}
$$

où la variété $X_{h}^{f_{h}}$ est le translaté de $X_{h}^{0}$ par la fonction $\bar{p}_{h}$. En vertu des théorèmes 3.1 et 4.2 , ce problème (4.16) admet une solution $\left(p_{h}, \lambda_{h}\right)$ et une seule dès que la condition (4.14) est satisfaite.

Remarque GÉNÉrale : Pour des raisons de simplicité, nous avons donné des conditions nécessaires et des conditions suffisantes pour que l'hypothèse de compatibilité (4.14) soit satisfaite en des propriétés des espaces $\mathscr{P}(K)$; il est clair que ces propriétés ne portent en fait que les espaces $\mathscr{P}(\hat{o} K)$, espaces des traces des fonctions de $\mathscr{P}(K)$.

\section{MAJORATION D'ERREUR DANS LA MÉTHODE DES ÉLÉMENTS FINIS HYBRIDES DUAUX}

Pour obtenir les majorations d'erreur, nous utilisons la technique du passage à l'élément fini de référence, comme dans [4] par exemple. Soit $\hat{O} \hat{x}_{1} \hat{x}_{2}$ un plan euclidien dit de référence et $\hat{K}$ le triangle de ce plan de sommets $(1,0),(0,1)$ et $(0,0)$. Pour tout triangle $K$ (non dégénéré) du plan euclidien $O x_{1} x_{2}$, il existe une application $F_{K}$ affine inversible telle que

$$
K=F_{K}(\hat{K}) \text {. }
$$

L'application linéaire tangente $\partial F_{K}$ est représentée par une matrice $2 \times 2$ constante, inversible; le jacobien $J_{K}$ de $F_{K}$ n'est autre que le rapport des aires des triangles $K$ et $\hat{K}$ :

$$
J_{K}=\left|\operatorname{det}\left(\partial F_{K}\right)\right|=\operatorname{Mes}(K) / \operatorname{Mes}(\hat{K}) .
$$

Nous notons $\mathscr{F}_{K}$ l'application qui à toute fonction scalaire $\hat{w}$ définie sur le triangle $\hat{K}$ associe la fonction $w=\mathscr{F}_{K}(\hat{w})$ définie sur $K$ par $w=\hat{w}_{0} F_{K}^{-1}$. De même $\mathscr{F}_{\partial K}$ est l'application qui à toute fonction scalaire $\hat{\mu}$ définie sur le bord $\partial \hat{K}$ associe la fonction $\mu=\mathscr{F}_{\partial K}(\hat{\mu})$ définie sur $\partial K$ par $\mu=\left.\hat{\mu}_{0}\left(F_{K}^{-1}\right)\right|_{\partial K}$. D'autre part, soit $\mathscr{G}_{K}$ l'application qui à toute fonction $\hat{q}$ définie sur le triangle $K$ et à valeurs dans $\mathbf{R}^{2}$ associe la fonction $q=\mathscr{G}_{K}(\hat{q})$ définie sur $K$ par

$$
q=J_{K}^{-1}\left(\partial F_{K} q\right) \circ F_{K}^{-1} .
$$

décembre 1976. 
Par convention d'écriture, on a désormais

$x=F_{K}(\hat{x}), \quad w=\mathscr{F}_{K}(\hat{w}), \quad \mu=\mathscr{F}_{\partial K}(\hat{\mu}), \quad q=\mathscr{G}_{K}(\hat{q})$,

LeMme $5.1:$ L'application $\mathscr{G}_{K}$ est un isomorphisme de l'espace $V(\hat{K})\left({ }^{3}\right)$. sur l'espace $V(K)$ tel que

$$
\begin{array}{r}
\forall w \in L_{2}(K), \quad \int_{K} w \operatorname{div} q d x=\int_{\hat{K}} \hat{w} \operatorname{div}_{\hat{x}} \hat{q} d \hat{x}, \\
\forall \mu \in H^{1 / 2}(\partial K), \quad \int_{\partial K} \mu v_{K} \cdot q d \gamma=\int_{\partial \hat{K}} \hat{\mu} v_{\hat{K}} \cdot \hat{q} d \hat{\gamma} .
\end{array}
$$

Puisque $d x=J_{K} d \hat{x}$, la propriété (5.4) s'écrit encore :

$$
\forall x \in K, \quad \operatorname{div} q(x)=J_{K}^{-1} \operatorname{div}_{\hat{x}} \hat{q}(\hat{x}) .
$$

Remarque 5.1 : La restriction de $\mathscr{G}_{K}$ au sous-espace $V^{0}(\hat{K})$ est l'application qui à la fonction $\hat{q}=\operatorname{rot}_{\hat{x}} \hat{w}$ associe la fonction $q=\operatorname{rot} w$.

REMARQue $5.2: \mathrm{La}$ démonstration donnée ci-après est valable dès que $F_{K}$ est un $C^{1}$-difféomorphisme de $\hat{K}$ sur $K$, ce qui permet l'extension aux éléments finis courbes. De même on n'utilise pas que $\hat{K}$ soit un triangle; on a la même démonstration avec $\hat{K} n$-simplexe de $\mathbf{R}^{n}, \hat{K}$ hypercube de $\mathbf{R}^{n}$, etc.

Démonstration du lemme 5.1: A l'aide d'un argument de densité, il suffit de vérifier la relation (5.4) pour toute fonction $w$ de classe $C^{1}$ dans $K$ et nulle sur le bord $\partial K$.

Soit $w$ une telle fonction. Par intégration par parties dans $K$, on a

$$
\int_{K} w \operatorname{div} q d x=-\int_{K} \operatorname{grad} w \cdot q d x
$$

On vérifie aisément la relation

$$
\forall x \in K, \quad{ }^{t}\left(\partial F_{K}\right) \operatorname{grad} w(x)=\operatorname{grad}_{\hat{x}} \hat{w}(\hat{x}),
$$

d'où

$$
\operatorname{grad} w(x) \cdot q(x)=J_{K}^{-1} \operatorname{grad} w(x) \cdot\left(\partial F_{K}\right) \hat{q}(\hat{x})=J_{K}^{-1} \operatorname{grad}_{\hat{x}} \hat{w}(\hat{x}) \cdot \hat{q}(\hat{x})
$$

( $\left.{ }^{3}\right)$ Dans le plan de référence $\hat{O} \hat{x}_{1} \hat{x}_{2}$, si $\hat{q}=\left(\hat{q}_{1}, \hat{q}_{2}\right)$,

$$
\operatorname{div}_{\hat{x}} \hat{q}=\frac{\partial \hat{q}_{1}}{\partial \hat{x}_{1}}+\frac{\partial \hat{q}_{2}}{\partial \hat{x}_{2}}
$$

$V(\hat{K})=\left\{q \in\left(L_{2}(\hat{K})\right) ; \operatorname{div}_{\hat{x}} \hat{q} \in L_{2}(\hat{K})\right\}$. 
soit puisque $d x=J_{K} d \hat{x}$ :

$$
\int_{K} \operatorname{grad} w \cdot q d x=\int_{\hat{K}} \operatorname{grad}_{\hat{x}} \hat{w} \cdot \hat{q} d \hat{x} .
$$

Par conséquent

$$
\int_{K} w \operatorname{div} q d x=-\int_{\hat{K}} \operatorname{grad}_{\hat{x}} \hat{w} \cdot \hat{q} d \hat{x},
$$

et on obtient en utilisant une intégration par parties dans $\hat{K}$ :

soit la relation $(5.4)$.

$$
\int_{K} w \operatorname{div} q d x=\int_{\hat{K}} \hat{w} \operatorname{div}_{\hat{x}} \hat{q} d \hat{x}
$$

Pour la seconde partie du lemme, soit $\mu$ un élément quelconque de l'espace $H^{1 / 2}(\partial K)$ : il existe une fonction $w \in H^{1}(K)$ dont la trace sur le bord $\partial K$ est la fonction $\mu$. La formule de Green (2.6) dans $K$ s'écrit :

$$
\int_{\partial K} \mu v_{K} \cdot q d \gamma=\int_{K} \operatorname{grad} w \cdot q d x+\int_{K} w \operatorname{div} q d x .
$$

On obtient en vertu de (5.4) et de (5.7) :

$$
\int_{\partial K} \mu v_{K} \cdot q d \gamma=\int_{\hat{K}} \operatorname{grad}_{\hat{x}} \hat{w} \cdot \hat{q} d \hat{x}+\int_{\hat{K}} \hat{w} \operatorname{div}_{\hat{x}} \hat{q} d \hat{x}
$$

et en utilisant la formule de Green (2.6) dans $\hat{K}$ :

$$
\int_{\partial K} \mu v_{K} \cdot q d \gamma=\int_{\partial \hat{K}} \hat{\mu} v_{\hat{K}} \cdot \hat{q} d \hat{\gamma}
$$

soit la relation $(5.5)$.

Soit d'autre part $\hat{R}$ l'application qui à une fonction scalaire $\hat{g}$ définie sur $\hat{K}$ associe la fonction $\hat{q}=\hat{R}(\hat{g})$ de composantes

$$
\left.\begin{array}{l}
\hat{q}_{1}\left(\hat{x}_{1}, \hat{x}_{2}\right)=-\int_{0}^{\hat{x}_{1}} \hat{g}\left(t, \hat{x_{2}}\right) d t \\
\hat{q}_{2}\left(\hat{x}_{1}, \hat{x}_{2}\right)=0 .
\end{array}\right\}
$$

Cette application $\hat{R}$ est linéaire continue de $L_{2}(\hat{K})$ dans $V(\hat{K})$ et telle que

$$
\forall \hat{g} \in L_{2}(\hat{K}), \quad \hat{R}(\hat{g}) \in V^{\hat{g}}(\hat{K}) .
$$

Nous définissons alors l'application $R_{K}$ par

$$
\forall g \in L_{2}(K), \quad R_{K}(g)=\mathscr{G}_{K}\left(\hat{R}\left(J_{K} \hat{g}\right)\right) .
$$

décembre 1976. 
Il résulte du lemme 5.1 que $R_{\mathrm{K}}$ est une application linéaire continue de $L_{2}(K)$ dans $V(K)$ telle que

$$
\forall g \in L_{2}(K), \quad R_{K}(g) \in V^{g}(K) .
$$

Dans le cas particulier qui nous intéresse ici, l'application $F_{K}$ est affine; on a alors l'expression simplifiée

$$
R_{K}(\mathrm{~g})=\partial F_{K} \hat{R}(\hat{g}) .
$$

La fonction $f_{h}$ ayant été définie à l'aide des relations (4.15), soit $\bar{p}_{h}$ la fonction de $\left(L_{2}(\Omega)\right)^{2}$ dont la restriction à tout triangle $K \in \mathscr{T}_{h}$ est

$$
\bar{p}_{h \mid K}=R_{K}\left(f_{h}\right)
$$

A l'aide de (5.11), on obtient

$$
\bar{p}_{h} \in X^{f_{h}} \text {. }
$$

On notera que la restriction de $\bar{p}_{h}$ à tout triangle $K$ est un élément de $\left(P_{m}(K)\right)^{2}$ dont la détermination explicite se ramène à un calcul évident de primitive dans le triangle $\hat{K}$.

Nous supposons désormais :

(i) la suite des triangulations $\mathscr{T}_{h}$ est régulière, i. e. il existe une constante $\sigma$ indépendante de $h$ telle que

$$
\forall K \in \mathscr{T}_{h}, \quad \sigma_{K} \leqq \sigma,
$$

où $\sigma_{K}$ est le rapport du diamètre du triangle $K$ au diamètre du cercle inscrit dans $K$;

(ii) pour tout triangle $K$ de $\mathscr{T}_{h}$, l'espace $\mathscr{P}(K)$ est construit à partir d'un espace de référence $\mathscr{P}(\hat{K})$ par

$$
\mathscr{P}(K)=\left\{q=\mathscr{G}_{K}(\hat{q}) ; \hat{q} \in \mathscr{P}(\hat{K})\right\},
$$

où $\mathscr{P}(\hat{K})$ est un espace de fonctions définies sur $\hat{K}$ tel que

$$
\left(P_{k}(\hat{K})\right)^{2} \subset \mathscr{P}(\hat{K}) \subset\left(P_{k^{\prime}}(\hat{K})\right)^{2} .
$$

Dans toute la suite, $C$ désigne diverses constantes indépendantes de $h$.

THÉORÈME 5.1 : On fait les hypothèses (5.14) et (5.15); on suppose en outre que

$$
\begin{aligned}
Z(\partial \hat{K})=\left\{\hat{\mu} \in P_{m}(\partial \hat{K}) ;\right. & \forall q \in \mathscr{P}(\hat{K}) \cap V^{-0}(\hat{K}) \\
& \left.\int_{\partial \hat{K}} \hat{\mu} v_{\hat{K}} \cdot \hat{q} d \hat{\gamma}=0\right\}=P_{0}(\partial \hat{K}) .
\end{aligned}
$$

Alors l'hypothèse de compatibilité uniforme (3.13) est satisfaite. 
Remarque 5.1 : Le lemme 4.1 et la remarque 4.1 fournissent des conditions suffisantes pour que l'hypothèse $(5.17)$ soit satisfaite.

Démonstration $d u$ théorème 5.1 : Montrons tout d'abord la minoration locale : il existe une constante $\beta>0$ indépendante de $h$ telle que pour tout triangle $K$ de $\mathscr{T}_{h}$ :

$$
\forall \mu \in P_{m}(\partial K), \quad \operatorname{Sup}_{q \in \mathscr{P}(K) \cap V^{0}(K)} \frac{\int_{\partial K} \mu v_{K} \cdot q d \gamma}{\|q\|_{0 . K}} \geqq \beta|\mu|_{1 / 2, \partial K} .
$$

Soit $\mu$ une fonction de $P_{m}(\partial K)$; la fonction $\hat{\mu}=\mathscr{F}_{\partial K}^{-1}(\mu)$ appartient à l'espace $P_{m} \partial(\hat{K})$. D'autre part à l'aide de l'hypothèse (5.15) et du lemme 5.1, on a

$$
\operatorname{Sup}_{q \in \mathscr{P}(K) \cap v^{0}(K)} \frac{\int_{\partial K} \mu v_{K} \cdot q d \gamma}{\|q\|_{0, K}}=\operatorname{Sup}_{\hat{q} \in \mathscr{P}(\hat{K}) \cap V^{0}(\hat{K})} \int_{\partial \hat{K}} \hat{\mu} v_{\hat{K}} \cdot \hat{q} \hat{d} \hat{\mathscr{G}_{K}(\hat{q}) \|_{0 . K}}
$$

D'après l'hypothèse $(5.15)$ on obtient par des majorations standard

$$
\forall \hat{q} \in\left(L_{2}(\hat{K})\right)^{2}, \quad\|\hat{q}\|_{0, \hat{K}} \leqq C_{1}\left\|\mathscr{G}_{K}(\hat{q})\right\|_{0, K}, \quad\left(C_{1}>0\right) .
$$

En vertu de l'hypothèse (5.17),

$$
\hat{\mu} \rightarrow \operatorname{Sup}_{\hat{q} \in \mathscr{P}(\hat{K}) \cap V^{0}(\hat{K})} \frac{\int_{\partial \hat{K}} \hat{\mu} v_{\hat{K}} \cdot \hat{q} d \hat{\gamma}}{\|\hat{q}\|_{0, \hat{K}}}
$$

définit une norme sur l'espace $P_{m}(\partial \hat{K}) / \mathbf{R}$ de dimension finie, on en déduit l'existence d'une constante $\hat{\beta}>0$ telle que

$$
\forall \hat{\mu} \in P_{m}(\hat{K}), \quad \operatorname{Sup}_{\hat{q} \in \mathscr{P}(\hat{K}) \cap V^{0}(\hat{K})} \frac{\int_{\partial \hat{K}} \hat{\mu} v_{\hat{K}} \cdot \hat{q} d \hat{\gamma}}{\|\hat{q}\|_{0, \hat{K}}} \geqq \hat{\beta}|\hat{\mu}|_{1 / 2, \partial \hat{K}}
$$

Enfin puisque pour toute fonction $\hat{v}$ de l'espace $H^{1}(\hat{K})$, on a

$$
C_{2}\left|\mathscr{F}_{K}(\hat{v})\right|_{1, K} \leqq|\hat{v}|_{1, \hat{K}} \quad\left(C_{2}>0\right)
$$

on déduit de la définition (2.25) :

$$
C_{2}|\mu|_{1 / 2, \partial K} \leqq|\hat{\mu}|_{1 / 2, \partial \hat{K}}
$$

Récapitulant les résultats, on obtient la minoration (5.18) avec pour constante $C=C_{1} \hat{\beta} C_{2}$.

décembre 1976. 
Soit alors $\mu_{h}$ un élément quelconque de l'espace $M_{h}$. Pour tout triangle $K \in \mathscr{T}_{h}$, nous pouvons donc trouver une fonction $\tilde{q}_{K} \in \mathscr{P}(K) \cap V^{0}(K)$ telle que

$$
\begin{gathered}
-\int_{\partial K} \mu_{h} v_{K}, \tilde{q}_{K} d \gamma \geqq \beta\left|\mu_{h}\right|_{1 / 2, \partial K}^{2} \\
\left\|\tilde{q}_{K}\right\|_{0, K}=\left|\mu_{h}\right|_{1 / 2, \partial K},
\end{gathered}
$$

Sojt $\tilde{q}_{h}$ la fonction de $\left(L_{2}(\Omega)\right)^{2}$ dont la restriction à tout $K \in \mathscr{T}_{h}$ est $\tilde{q}_{K}$; cette fonction $\tilde{q}_{h}$ appartient à l'espace $X_{h}^{0}$. Elle vérifie d'une part

$$
b\left(\tilde{q}_{h}, \mu_{h}\right)=-\sum_{K \in \mathscr{T}_{h}} \int_{\partial K} \mu_{h} v_{K} \cdot \tilde{q}_{K} d \gamma \geqq \beta \sum_{K \in \mathscr{T}_{h}}\left|\mu_{h}\right|_{1 / 2, \partial K}^{2}
$$

soit en vertu du lemme 2.1 :

$$
b\left(\tilde{q}_{h}, \mu_{h}\right) \geqq \beta\left\|\mu_{h}\right\|_{M}^{2} .
$$

Elle vérifie d'autre part

soit

$$
\left\|\tilde{q}_{h}\right\|_{X}^{2}=\sum_{K \in \mathscr{T}_{h}}\left\|\tilde{q}_{K}\right\|_{0, K}^{2}=\sum_{K \in \mathscr{T}_{h}}\left|\mu_{h}\right|_{1 / 2, \partial K}^{2}
$$

$$
\left\|\tilde{q}_{h}\right\|_{X}=\left\|\mu_{h}\right\|_{M} .
$$

On a ainsi trouvé une fonction $\tilde{q}_{h}$ de l'espace $X_{h}^{0}$ qui satisfait

$$
b\left(\tilde{q}_{h}, \mu_{h}\right) \geqq \beta\left\|\tilde{q}_{h}\right\|_{X}\left\|\mu_{h}\right\|_{M} ; \quad\left\|\tilde{q}_{h}\right\|_{X}=\left\|\mu_{h}\right\|_{M}
$$

ce qui démontre la relation (3.13).

On notera que dans le cas $k=k^{\prime}=m-1, m$ pair, l'hypothèse de compatibilité (simple) (3.12) est satisfaite; or dans cette situation l'hypothèse (5.17) n'est pas satisfaite. A l'aide d'un contre-exemple, nous avons montré dans [14] que l'hypothèse de compatibilité uniforme (3.13) n'est pas satisfaite dans ce cas.

Les théorèmes 3.1 et 5.1 permettent de majorer l'erreur commise sur la solution $(p, \lambda)$ à l'aide des erreurs d'approximation dans les espaces $X_{h}^{0}$ et $M_{h}$.

Lemme 5.2 : On fait les hypothèses (5.14) et (5.15); on suppose en outre que

$$
\left(P_{m-1}(\hat{K})\right)^{2} \subset \mathscr{P}(\hat{K}) \text {. }
$$

Alors, pour toute fonction $\tilde{p} \in X^{0} \cap \prod_{K \in \mathscr{T}_{h}}\left(H^{m}(K)\right)^{2}$, on a la majoration

$$
\operatorname{Inf}_{q_{h} \in X_{h}^{0}}\left\|\tilde{p}-q_{h}\right\|_{X} \leqq C h^{m}\left\{\sum_{K \in \mathscr{T}_{h}}|\tilde{p}|_{m, K}^{2}\right\}^{1 / 2},
$$

où $C$ est une constante indépendante de $h$. 
Démonstration : Soit $K$ un triangle de $\mathscr{T}_{h}$, puisque div $\tilde{p}=0$ dans $K$, en introduisant une fonction courant : $\tilde{p}=\operatorname{rot} \tilde{w}$, on a

$$
\operatorname{Inf}_{q \in\left(P_{m-1}(K)\right)^{2} \cap V^{0}(K)}\|\tilde{p}-q\|_{0, K}=\operatorname{Inf}_{w \in P_{m}(K)}|\tilde{w}-w|_{1, K}
$$

Par un argument classique, on a

$$
\operatorname{Inf}_{w \in P_{m}(K)}|\tilde{w}-w|_{1, K} \leqq C h^{m}|\tilde{w}|_{m+1, K},
$$

ce qui conduit, après avoir remarqué que $|\tilde{w}|_{m+1, K}=|\tilde{p}|_{\mid m, K}$, à la majoration dans $K$ :

$$
\operatorname{Inf}_{q \in\left(P_{m-1}(K)\right)^{2} \cap V^{\circ}(K)}\|\tilde{p}-q\|_{0, K} \leqq C h^{m}|\tilde{p}|_{m, K}
$$

A l'aide de l'hypothèse (5.18), on a

$$
\begin{aligned}
\underset{q_{h} \in X_{h}^{0}}{\operatorname{Inf}}\left\|\tilde{p}-q_{h}\right\|_{X} & =\operatorname{Inf}_{q_{h} \in X_{h}^{0}}\left\|\tilde{p}-q_{h}\right\|_{0, \Omega} \\
& \leqq \sum_{K \in \mathscr{T}_{h}}\left\{\underset{q \in\left(P_{m-1}(K)\right)^{2} \cap V^{0}(K)}{\operatorname{Inf}}\|\tilde{p}-q\|_{0, K}^{2}\right\}^{1 / 2},
\end{aligned}
$$

soit avec la majoration (5.20) :

$$
\operatorname{Inf}_{q_{h} \in X_{h}^{0}} \| \tilde{p}-\left.q_{h}\right|_{x} \leqq C h^{m}\left\{\sum_{K \in \mathscr{T}_{h}}|\tilde{p}|_{m, K}^{2}\right\}^{1 / 2} .
$$

Lemme 5.3 : On fait l'hypothèse (5.14). Pour toute fonction $\lambda \in M$ telle que la variété $\mathscr{H}(\lambda) \cap H^{m+1}(\Omega)$ soit non vide, on a la majoration

$$
\operatorname{Inf}_{\mu_{h} \in \mathscr{T}_{h}}\left\|\lambda-\mu_{h}\right\|_{M} \leqq C h^{m}|u|_{m+1, \Omega},
$$

où $u$ est une fonction (quelconque) de $\mathscr{H}(\lambda) \cap H^{m+1}(\Omega)$.

Démonstration : Soit $u \in \mathscr{H}(\lambda) \cap H^{m+1}(\Omega)$; puisque nous sommes en dimension 2 d'espace et comme $m$ est un entier $\geqq 1$, les théorèmes d'injection de Sobolev impliquent que la fonction $u$ est continue sur $\bar{\Omega}$. Il est alors bien connu qu'il existe une fonction $\tilde{v}_{h}$ continue sur $\bar{\Omega}$, nulle sur $\Gamma$, polynômiale de degré $\leqq m$ dans tout triangle $K$ et telle que

$$
\left|u-\tilde{v}_{h}\right|_{1, \Omega} \leqq C h^{m}|u|_{m+1, \Omega}
$$

Soit $\tilde{\mu}_{h}$ la fonction de $\prod_{K \in \mathscr{T}_{h}}\left(L_{2}(\partial K)\right)$ définie par

$$
\forall K \in \mathscr{T}_{h}, \quad \tilde{\mu}_{h}=\tilde{v}_{h} \quad \text { sur } \partial K .
$$

Il est clair que cette fonction $\tilde{\mu}_{h}$ appartient à l'espace $M_{h}$; de plus $u-\tilde{v}_{h}$ est élément de $\mathscr{H}\left(\lambda-\tilde{\mu}_{h}\right)$. On en déduit

$$
\left\|\lambda-\tilde{\mu}_{h}\right\|_{M} \leqq\left|u-\tilde{v}_{h}\right|_{1, \Omega} \leqq C h^{m}|u|_{m+1, \Omega},
$$

décembre 1976. 
d'où la majoration

$$
\operatorname{lnf}_{\mu_{h} \in M_{h}}\left\|\lambda-\mu_{h}\right\|_{M} \leqq C h^{m}|u|_{m+1, \Omega}
$$

Nous pouvons à présent énoncer le résultat fondamental :

THÉORÈme 5.2 : On fait les hypothèses (5.14) et (5.15); on suppose en outre

$$
Z(\partial \hat{K})=P_{0}(\partial \hat{K})
$$

et

$$
\left(P_{m-1}(\hat{K})\right)^{2} \subset \mathscr{P}(\hat{K}) .
$$

Le problème (4.16) admet une solution $\left(p_{h}, \lambda_{h}\right)$ et une seule.

Si

$$
u \in H^{m+1}(\Omega), \quad p \in\left(H^{m}(\Omega)\right)^{2}, \quad \operatorname{div} p \in H^{m}(\Omega),
$$

où $u$ est la solution du problème (2.2) et $(p, \lambda)$ la solution du problème (2.31), il existe une constante $C$ indépendante de $h$ telle que

$\mid i p-p_{h}\left\|_{X}+\right\| \lambda-\lambda_{h} \|_{M} \leqq C h^{m}\left\{|u|_{m+1, \Omega}+|p|_{m, \Omega}+|\operatorname{div} p|_{m, \Omega}\right\}$.

Démonstration : Nous introduisons la solution $u^{*}$ du problème analogue à (2.2) obtenue avec pour second membre non plus la fonction $f$ mais son approximation $f_{h}$ :

$$
\mathscr{A} u^{*}=f_{h} \quad \text { dans } \Omega, \quad u^{*}=0 \quad \text { sur } \Gamma \text {. }
$$

De même soit $\left(p^{*}, \lambda^{*}\right)$ la solution du problème obtenu en substituant $f_{h}$ à $f$ dans la formulation (2.31). On a

$$
\left|u-u^{*}\right|_{1, \Omega} \leqq C\left\|f-f_{h}\right\|_{0, \Omega},
$$

d'où en utilisant les caractérisations données au théorème 2.2 :

$$
\left\|p-p^{*}\right\|_{0, \Omega}+\left\|\lambda-\lambda^{*}\right\|_{M} \leqq C\left\|f-f_{h}\right\|_{0, \Omega} \text {. }
$$

D'après le théorème 5.1 la condition de compatibilité uniforme (3.13) est satisfaite, de sorte que l'application du théorème 3.2 fournit la majoration

$$
\begin{aligned}
& \left\|p^{*}-p_{h}\right\|_{0, \Omega}+\left\|\lambda^{*}-\lambda_{h}\right\|_{M} \\
& \quad \leqq C\left\{\underset{q_{h} \in X_{h}^{f_{h}}}{\operatorname{Inf}}\left\|p^{*}-q_{h}\right\|_{0, \Omega}+\underset{\mu_{h} \in M_{h}}{\operatorname{Inf}}\left\|\lambda^{*}-\mu_{h}\right\|_{M}\right\} .
\end{aligned}
$$

Soit $\bar{p}$ la fonction de $\left(L_{2}(\Omega)\right)^{2}$ dont la restriction à tout triangle $K \in \mathscr{T}_{h}$ est

$$
\left.\bar{p}\right|_{K}=R_{K}(f) \text {. }
$$


A l'aide de (5.14), on a

$$
\left\|\bar{p}-\bar{p}_{h}\right\|_{0, \Omega}=\left\{\sum_{K \in \mathscr{T}_{h}}\left\|R_{K}\left(f-f_{h}\right)\right\|_{0, K}^{2}\right\}^{1 / 2} \leqq C h\left\|f-f_{h}\right\|_{0, \Omega}
$$

Des majorations (5.23), (5.24) et (5.25) on déduit

$$
\begin{aligned}
& \left\|p-p_{h}\right\|_{0, \Omega}+\left\|\lambda-\lambda_{h}\right\|_{M} \\
& \quad \leqq C\left\{\left\|f-f_{h}\right\|_{0, \Omega}+\operatorname{Inf}_{q_{h} \in X_{h}^{0}}\left\|\tilde{p}-q_{h}\right\|_{0, \Omega}+\operatorname{Inf}_{\mu_{h} \in M_{h}}\left\|\lambda-\mu_{h}\right\|_{M}\right\},
\end{aligned}
$$

où $\tilde{p}=p-\bar{p}$ appartient au sous-espace $X^{0} ;$ comme $\operatorname{div}\left(p-p_{h}\right)+f-f_{h}=0$ dans tout triangle $K$, on a ainsi

$$
\begin{aligned}
& \left\|p-p_{h}\right\|_{X}+\left\|\lambda-\lambda_{h}\right\| \\
& \quad \leqq C\left\{\left\|f-f_{h}\right\|_{0, \Omega}+\operatorname{Inf}_{q_{h} \in X_{h}^{0}}\left\|\tilde{p}-q_{h}\right\|_{0, \Omega}+\operatorname{Inf}_{\mu_{h} \in M_{h}}\left\|\lambda-\mu_{h}\right\|_{M}\right\} .
\end{aligned}
$$

A l'aide des lemmes 5.2, 5.3 et de la majoration standard obtenue à partir de (4.15):

$$
\left\|f-f_{h}\right\|_{0, \Omega} \leqq C h^{m}|f|_{m, \Omega},
$$

on trouve,

$$
\begin{aligned}
& \left\|p-p_{h}\right\|_{X}+\left\|\lambda-\hat{\lambda}_{h}\right\|_{M} \\
& \quad \leqq C h^{m}\left\{|u|_{m+1, \Omega}+\left(\sum_{K \in \mathscr{T}_{h}}|\tilde{p}|_{m, K}^{2}+|\operatorname{div} p|_{m, \Omega}\right\} .\right.
\end{aligned}
$$

Il reste à majorer la quantité $\left(\sum_{K \in \mathscr{T}_{h}}|\tilde{p}|_{m, K}^{2}\right)^{1 / 2}$; on a à l'aide de l'inégalité triangulaire :

$$
\left\{\sum_{K \in \mathscr{T}_{h}}|\tilde{p}|_{m, K}^{2}\right\}^{1 / 2} \leqq \sqrt{2}\left\{|p|_{m, \Omega}+\left\{\sum_{K \in \mathscr{T}_{h}}\left|R_{K}(f)\right|_{m, K}^{2}\right\}^{1 / 2}\right\}
$$

On remarque que si $\hat{q}=\hat{R}(\hat{g})$, on a d'après la définition (5.8) :

$$
\frac{\partial^{m} \hat{q}_{1}}{\partial \hat{x}_{1}^{\alpha} \partial \hat{x}_{2}^{m-\alpha}}\left(\hat{x}_{1}, \ddot{x}_{2}\right)= \begin{cases}-\int_{0}^{\hat{x}_{1}} \frac{\partial^{m} \hat{g}}{\partial \hat{x}_{2}^{m}}\left(t, \hat{x}_{2}\right) d t & \text { si } \quad \alpha=0, \\ -\frac{\partial^{m-1} \hat{g}}{\partial \hat{x}_{1}^{\alpha} \partial \hat{x}_{2}^{m-\alpha-1}}\left(\hat{x}_{1}, \hat{x}_{2}\right) & \text { si } \quad \alpha>0\end{cases}
$$

d'où la majoration dans le triangle $\hat{K}$ :

On en déduit

$$
|\hat{R}(\hat{g})|_{m, \hat{K}} \leqq|\hat{g}|_{m-1, \hat{K}}+|\hat{g}|_{m, \hat{K}}
$$

$$
\left|R_{K}(f)\right|_{m, K} \leqq C h J_{K}^{1 / 2} \frac{1}{h^{m}}\left\{\left|\mathscr{F}_{K}^{-1}(f)\right|_{m-1, \hat{K}}+\left|\mathscr{F}_{K}^{-1}(f)\right|_{m, \hat{K}}\right\}
$$

décembre 1976. 
ce qui donne dans le triangle $K$ :

$$
\left|R_{K}(f)\right|_{m, K} \leqq C\left\{|f|_{m-1, K}+h|f|_{m, K}\right\} \leqq C\left\{|p|_{m, K}+h|\operatorname{div} p|_{m, K}\right\} .
$$

Finalement, on obtient la majoration

$$
\left\{\sum_{K \in \mathscr{T}_{h}}|\tilde{p}|_{m, K}^{2}\right\}^{1 / 2} \leqq C\left\{|p|_{m, \Omega}+h|\operatorname{div} p|_{m, \Omega}\right\} .
$$

Reportant ce résultat dans (5.27), on a

$$
\left\|p-p_{h}||_{X}+\right\| \lambda-\lambda_{h} \|_{M} \leqq C\left\{|u|_{m+1, \Omega}+|p|_{m, \Omega}+|\operatorname{div} p|_{m, \Omega}\right\}
$$

ce qui démontre le théorème 5.2.

En conclusion lorsque $m$ est impair le choix le plus simple de l'espace $X_{h}^{0}$ conduisant à une méthode d'ordre $m$ est obtenu par

$$
\forall K \in \mathscr{T}_{h}, \quad \mathscr{P}(K)=\left(P_{m-1}(K)\right)^{2} .
$$

Lorsque $m$ est pair, le choix (5.29) ne conduit pas à une méthode d'ordre $m$; pour obtenir l'ordre $m$ il faut dans ce cas que les espaces $\mathscr{P}(K)$ contiennent outre $\left(P_{m-1}(K)\right)^{2}$ une fonction convenablement choisie ( $c f$. remarque 4.1).

Signalons enfin que la technique utilisée tout au long des démonstrations s'adapte sans difficulté nouvelle au cas où le domaine $\Omega$ est muni d'une quadrangulation à l'aide de parallélogrammes.

\section{BIBLIOGRAPHIE}

1. F. BRÉZZI, On the Existence, Uniqueness and Approximation of Saddle-point Problems Arising from Lagrangian Multipliers, R.A.I.R.O., R 2, 1974, p. 129 à 151 .

2. F. BRÉzZI, Sur une méthode hybride pour l'approximation du problème de la torsion d'une barre élastique, Istituto Lombardo (Rend. Sc.), A 108, 1974, p. 274 à 300 .

3. P.-G. Ciarlet, Numerical Analysis of the Finite Element Method (à paraître chez North-Holland).

4. P.-G. Ciarlet et P.-A. Raviart, General Lagrange and Hermite Interpolation in $\mathbf{R}^{n}$ with Applications to Finite Element Methods, Arch. Rat. Mech. Anal., vol. 46, 1972, p. 177 à 189.

5. F. De Veubeke, Variational Principles and the Patch Test, Int. Num. Meth. Eng., vol. 8, 1974, p. 783 à 801 .

6. J.-L. Lions et E. MAGenes, Problèmes aux limites non homogènes et applications, vol. 1, Dunod, Paris, 1968.

7. J. NEČAS, Les méthodes directes dans la théorie des équations elliptiques, Éditions de l'Académie Tchécoslovaque de Sciences, Prague, 1967.

8. T. H. H. PIAN, Formulations of Finite Element Methods for Solid Continua, Recent Advances in Matrix Methods of Structural Analysis and Design, (R. H. Gallagher, Y. Yamada, J. T. Oden Ed.), The Univ. of Alabama Press, 1971, p. 49 à 83 . 
9. T. H. H. PIAN, Finite Element Formulation by Variational Principles with Relaxed Continuity Requirements, The Mathematical Foundations of the Finite Element Method (A. K. Aziz, Ed.), Academic Press, 1972, p. 671 à 687.

10. T. H. H. Pian et P. Tong, Basis of Finite Element Methods for Solid Continua, Int. J. Numer. Meth. Eng., vol. 1, 1969, p. 3 à 28.

11. P. A. RaviaRT, Hybrid Finite Element Methods for solving 2nd Order Elliptic Equations, Conference on Numerical Analysis, Royal Irish Academy, Dublin, 1974.

12. P.-A. Raviart et J.-M. Thomas, Primal Hybrid Finite Element Methods for 2nd Order Elliptic Equations (à paraître).

13. G. Strang et G. FIX, An Analysis of the Finite Element Method, Prentice Hall, 1973.

14. J.-M. Thomas, Éléments finis de type PIAN, Séminaire Ciarlet-GlowinskiRaviart, Université Pierre-et-Marie-Curie, Paris, 1974.

15. J.-M. Thомаs, Méthode des éléments finis équilibre, Journées éléments finis. Rennes, 12,13 et 14 mai 1975. 